\title{
Development of a Flat-Panel and Resistor-Type Photomultiplier Tube System for High Position-Resolution Two-Dimensional Neutron Detector ${ }^{*}$
}

\author{
Setsuo SATOH \\ High Energy Accelerator Research Organization (KEK), 1-1 Oho, Tsukuba, Ibaraki 305-0801, Japan
}

(Received 28 May 2017 / Accepted 21 December 2017)

\begin{abstract}
A flat-panel and resistor-type photomultiplier tube (FRP) system, which is a two-dimensional positionsensitive neutron detector, has been developed. The FRP detector consists of a scintillator, a multi-anode photomultiplier tube (MA-PMT), a resistor network board, and an FRP amplifier and a readout circuit which is similar to that for the old resistor type photomultiplier tube (RPMT) system. Therefore, the FRP system uses the same readout circuit of the RPMT system. Two types of FRP detector are available. One is the FRP4 detector, which consists of four MA-PMTs with a detection area of $10-\mathrm{cm}$ square. The position resolution due to irradiation of neutron beam with approximately $0.5-\mathrm{mm}$ diameter is $1.26 \mathrm{~mm}$ in the $\mathrm{X}$ axis and $1.61 \mathrm{~mm}$ in the $\mathrm{Y}$ axis. The other one is the FRP1, which consists of one MA-PMT with a detection area of 5-cm square. The position resolution due to irradiation of a neutron beam with approximately $0.5-\mathrm{mm}$ diameter is $0.83 \mathrm{~mm}$ in the $\mathrm{X}$ axis and $0.89 \mathrm{~mm}$ in the $\mathrm{Y}$ axis. The FRP detector can be used for high position-resolution and transmission experiments of a compact neutron source.
\end{abstract}

(c) 2018 The Japan Society of Plasma Science and Nuclear Fusion Research

Keywords: FRP system, RPMT system, two-dimensional neutron detector, high position-resolution neutron detector, compact neutron source

DOI: $10.1585 /$ pfr. 13.2405056

\section{Introduction}

Neutron-scattering experiments are indispensable in the structural analysis of various forms of condensed matter and in the development of advanced materials. These days, industrial applications have been increasing. Therefore, large-scale experimental facilities where these experiments can be performed are being constructed globally. In Japan, the Materials and Life Science Experimental Facility (MLF) in the Japan Proton Accelerator Research Complex was built in 2008 and is currently in operation. In many neutron experiments there, ${ }^{3} \mathrm{He}$ gas detector is used for high reliability [1]. The ${ }^{3} \mathrm{He}$ gas detector is a proportional counter, which hardly senses gamma rays, can easily separate neutrons from noise, and has high neutrondetection efficiency. However, its position resolution in the absence of special processing is limited to approximately $8 \mathrm{~mm}$. Therefore, the data acquisition group at the Neutron Science Laboratory (KENS) in the High-Energy Accelerator Research Organization (KEK) developed a resistortype photomultiplier tube (RPMT) system [2]. The RPMT system is readily used in neutron experiments that require a position resolution of $1 \mathrm{~mm}$ or less. However, because the production of photomultiplier tubes (PMTs) for an RPMT system was stopped in March 2016, a flat-panel and

author'se-mail: setsuo.satoh@kek.jp

*) This article is based on the presentation at the Conference on Laser Energy Science / Laser and Accelerator Neutron Sources and Applications 2017. resistor-type PMT (FRP) neutron detector system has been developed as a substitute. Because the readout-circuit in Ref. [1] is used, the time-of-flight resolution of the FRP system is $25 \mathrm{~ns}$. Its neutron-detection efficiency depends on a scintillator. In thermal neutrons, approximately $20 \%$ are obtained by $0.25-\mathrm{mm}$-thick $\mathrm{ZnS} /{ }^{6} \mathrm{LiF}$, and approximately $80 \%$ are obtained by a $1-\mathrm{mm}$-thick ${ }^{6} \mathrm{Li}$ glass scintillator.

\section{RPMT System}

The RPMT detector consists of a two-dimensional (2D) PMT (made by Hamamatsu Photonics Co.) and a neutron scintillator. Figure 1 shows the PMTs for the RPMT detectors. They are R3292 (9-cm-diameter detection area) with a 5-in size and R2486 (5-cm-diameter detection area) with a 3 -in size. When 0.45 -mm-thick $\mathrm{ZnS} /{ }^{6} \mathrm{LiF}$ is used as the neutron scintillator, the position resolution is $1 \mathrm{~mm}$ or less, and the detection efficiency is approximately $30 \%$ under thermal neutron. When a 1-mm-thick ${ }^{6} \mathrm{Li}$ glass (GS20) is used as the neutron scintillator, the position resolution is approximately $2 \mathrm{~mm}$, and the detection efficiency is approximately $80 \%$ under thermal neutron.

Figure 2 shows the circuit diagram of an R3292 data sheet [3] for the RPMT detector. The light from a neutron scintillator is converted to electrons in the photo cathode, which is obtained from the anode wires that are indepen- 


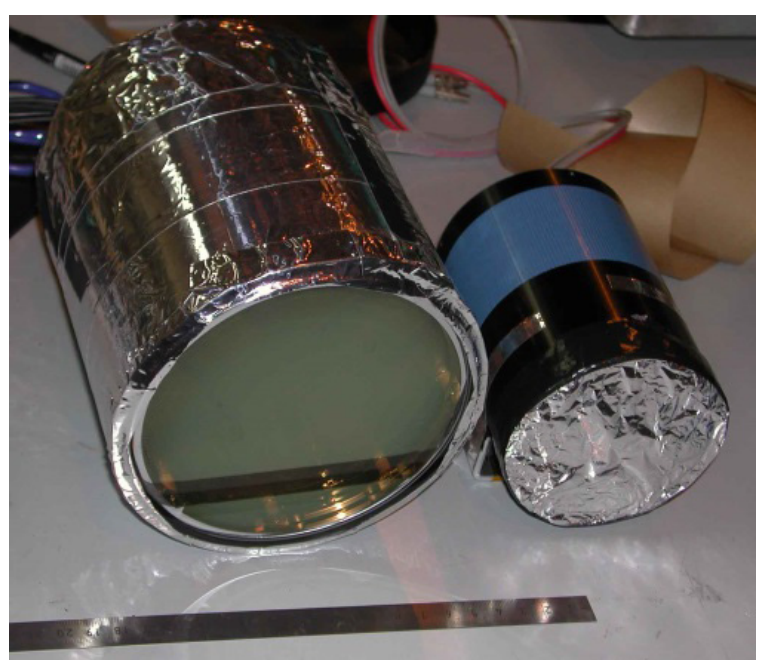

Fig. 1 RPMT tubes with 5- and 3-in sizes.

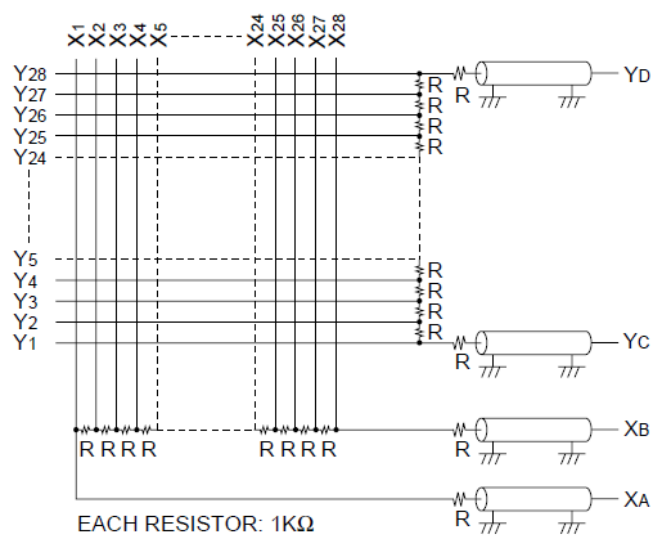

Fig. 2 Circuit diagram of the R3292 anode wires.

dently stretched in the $\mathrm{X}$ and $\mathrm{Y}$ axes. The outputs of the wires are connected to resistor networks. The position is obtained using the charge-division method. The number of R3292 wires in each axis is 28 . In detail, the light of the neutron scintillator is converted into electrons and reaches the anode wires as electric charge while being amplified. This charge is also diffused and absorbed by three or more wires, and the amount of each charge is larger at the center of the light. These charges with their corresponding weights are supplied to the resistor network. The position of the light center yields better resolution than the number of wires.

Figure 3 shows the principle diagram of the chargedivision method. Because both side voltages of the resistor must be kept at $0 \mathrm{~V}$ by the charge amplifiers, the charge on the resistor is divided proportional to the reciprocal of the resistance in each amplifier. Therefore, the position of the charge in the resistor is obtained using the ratio of the voltage outputs by the amplifiers at both sides. Assuming that the charge amounts obtained by the respective ampli-

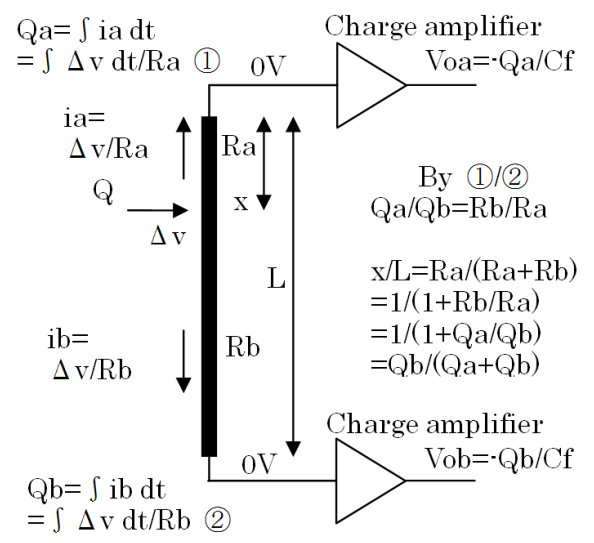

Fig. 3 Charge-division method.

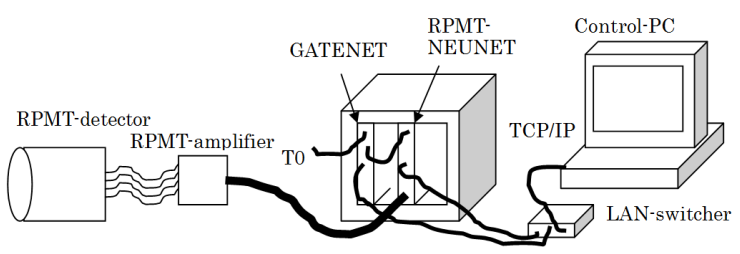

Fig. 4 RPMT system.

fiers are $Q a$ and $Q b$, the charge generated at position $x$ is a relational expression of $x / L=Q b /(Q a+Q b)$, where $L$ is the total resistor length. When the positions on the $\mathrm{X}$ and $\mathrm{Y}$ axes are independently obtained at the same time, as shown in Fig. 2, they are recognized as 2-D position data.

Figure 4 shows the configuration diagram of the RPMT system. The readout circuit of the RPMT system employs a NEUNET circuit[1] for the ${ }^{3} \mathrm{He}$ gas position-sensitive detectors (PSDs). Approximately 1,000 NEUNET circuits are employed in the MLF, which are the default standard. Because the NEUNET circuit outputs event data, many histogram data can be reconstructed from many perspectives after the experiment.

The NEUNET circuit has eight charge-division functions, converts the outputs of the eight PSDs to digital data, and generates event data. The RPMT system uses the two functions to obtain the positions of the $\mathrm{X}$ and $\mathrm{Y}$ axes. When they are obtained at the same time, the positions are recognized as 2-D data.

\section{FRP System}

Instead of using a specially designed PMT for the RPMT system, the FRP system employs a multi-anodetype PMT (MA-PMT), which is used in many cases in these days, especially in medical applications. Figure 5 shows the MA-PMT of an $8 \times 8$ array and a resistor array circuit diagram, which is attached behind the MA-PMT. Because the MA-PMTs have a square shape, they can easily be installed side by side. 

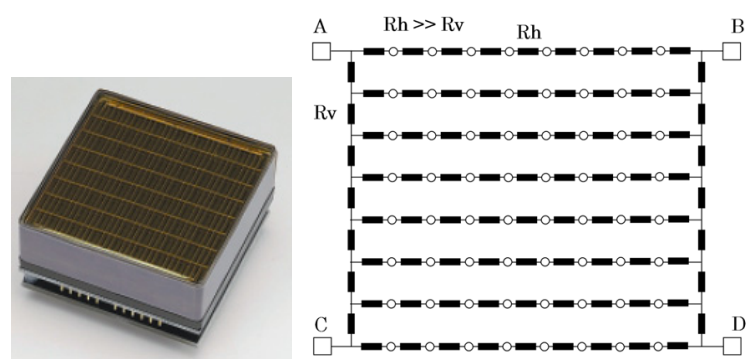

Fig. 5 MA-PMT and a resistor array circuit for an FRP detector.
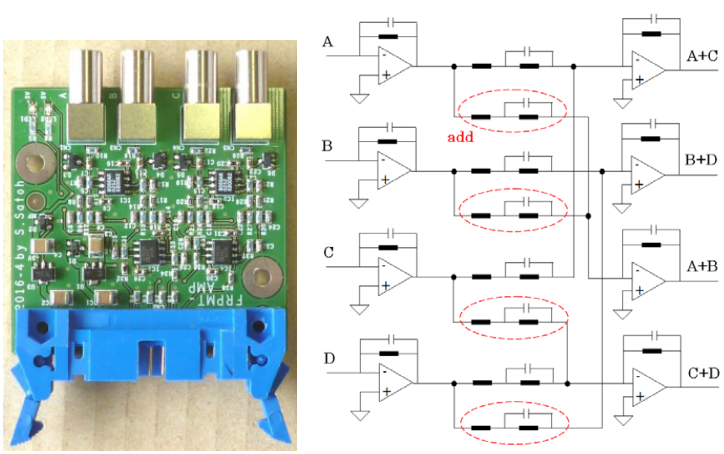

Fig. 6 FRP amplifier and its circuit diagram.

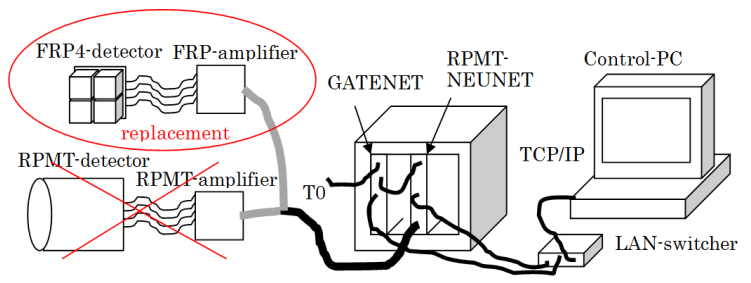

Fig. 7 FRP system.

Figure 6 shows the appearance and circuit diagram of the FRP amplifier. $\mathrm{XA}=\mathrm{A}+\mathrm{C}, \mathrm{XB}=\mathrm{B}+\mathrm{D}, \mathrm{YC}=\mathrm{A}+\mathrm{B}$ and $\mathrm{YD}=\mathrm{C}+\mathrm{D}$, where $\mathrm{XA}, \mathrm{XB}, \mathrm{XC}$, and $\mathrm{YD}$ are the outputs of the RPMT, and A, B, C, and D are the outputs of the FRP resistor network. Several circuits are added to the FRP amplifier to obtain signals equivalent to those of the RPMT amplifier.

Figure 7 shows the configuration of the FRP system. Because the FRP amplifier outputs signals similar to the RPMT amplifier, the FRP system is able to use the same readout circuit of the RPMT system. However, although the MA-PMT can perform parallel processing, it has a low count rate of approximately 10,000 counts per second because it uses an encoding system connected to the resistor network.
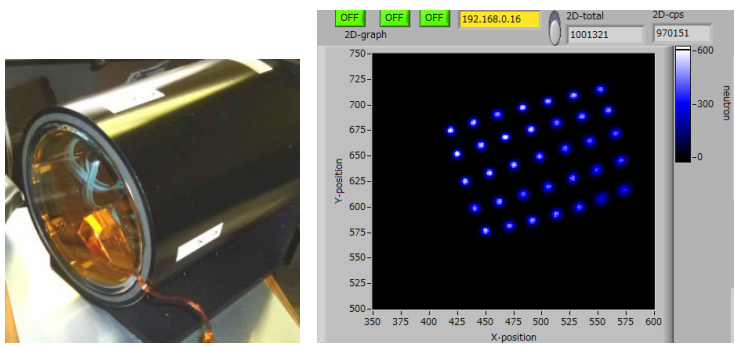

Fig. 8 RPMT with a LED array and 2D graph.
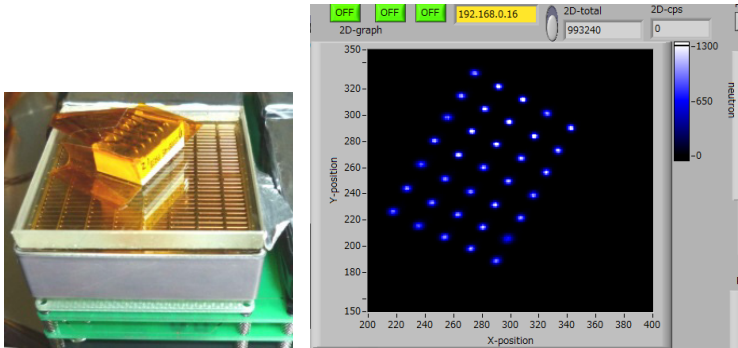

Fig. 9 FRP with a LED array and 2-D graph.

\section{Comparison of the RPMT and FRP Systems}

Figures 8 and 9 show the performance comparison of the RPMT and FRP detectors. The 2-D graphs are obtained by attaching a LED test circuit.

In the LED test circuit, $5 \times 7$ array LEDs are lighted, which match with the light emission and pulse width of the neutron scintillator. Among these LEDs, only one is lighted at a single time to test the encoding system. Because using a neutron source is difficult, the LED test circuit is indispensable for adjustment of a detector system such as 2-D PMTs or silicon photodetectors.

The 2-D graph of the RPMT detector shows a round distortion against the row of the LEDs. The graph of the FRP detector shows lesser distortion than that of the RPMT detector. One of the reasons for this is that, the quantum efficiency of the RPMT detector is $23 \%$ and that of the FRP detector increases to $33 \%$. Therefore, by receiving the same quantity of light, the FRP detector obtains $43 \%$ larger signal than the RPMT detector. Another reason is the increase in amplification. Although, the RPMT detector is approximately $1.25 \times 10^{5}$, the FRP is approximately $1.5 \times 10^{6}$, and the range that can be amplified with low noise is extended to more than 10 times.

\section{FRP System Data}

The maximum size of a commercial base MA-PMT is limited to $5-\mathrm{cm}$ square. However, many experiments require $10-\mathrm{cm}$ square or more in the detection area. For many neutron-scattering experiments and transmission imaging, 

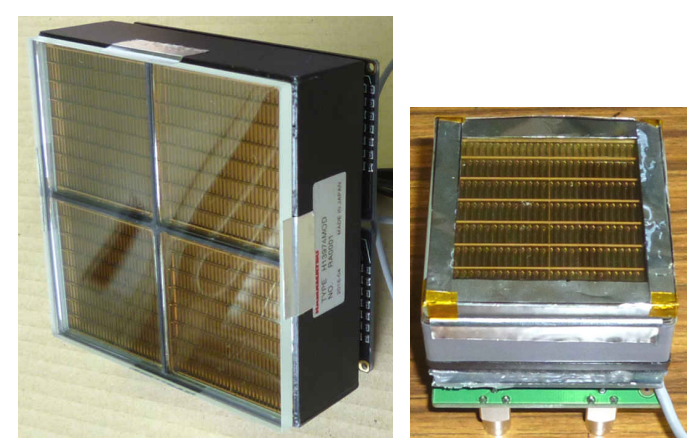

Fig. 10 FRP4 and FRP1 detectors.

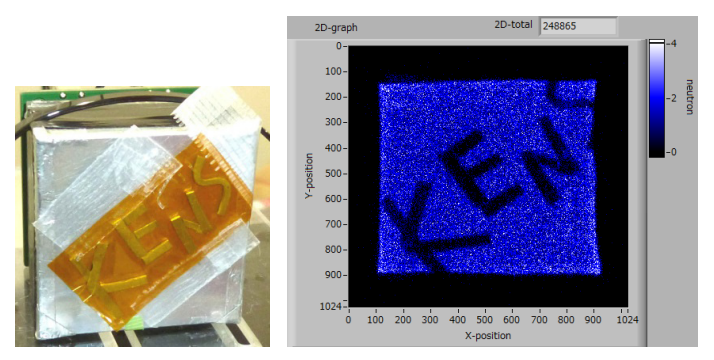

Fig. 11 Cd characters and 2-D graph of FRP1.

fine position resolution is desirable with a detection area that is as wide as possible. Because the RPMT detector has a circular detection area with a diameter of $9 \mathrm{~cm}$, a detection area of $10-\mathrm{cm}$ square is preferable as a substitute detector. Therefore, Hamamatsu Photonics Co. has developed an FRP4 detector that combines four MA-PMTs as H13974MOD. However, because the distortion at the joint of the FRP is large, KEK has developed an FRP1 detector with one MA-PMT, which has a narrow detection area of 5 -cm square or less. Figure 10 shows the inner parts of the FRP4 and FRP1 detectors.

\subsection{FRP1 detector}

The maximum deviation error of the measurement position when irradiated at 5-mm intervals is approximately $0.4 \mathrm{~mm}$. The position resolution due to irradiation of neutron beam with approximately $0.5-\mathrm{mm}$ diameter is $0.83 \mathrm{~mm}$ in the $\mathrm{X}$ axis and $0.89 \mathrm{~mm}$ in the $\mathrm{Y}$ axis.

Figure 11 shows the attachment of the $\mathrm{Cd}$ characters and the 2-D graph. Because the 0.5-mm-thick Cd almost completely shields the neutrons under $0.5 \mathrm{eV}$, it is used to intuitively evaluate the sharpness and distortion of a 2-D detector. Because the edge of the detector has a large distortion, the edge portion is covered with aluminum foil like the FRP1 detector in Fig. 10, and the detector has a detection area of $3.5 \times 3.5 \mathrm{~cm}^{2}$. To use the center-of-gravity calculation, a 5-mm-thick diffusion glass is installed, which diffuses the light to more than three anodes. Because the left and right sides of the 2-D graph are slightly dented, adjustment of the network resistance value is needed.

\section{Conclusion}

In this study, square-area detectors with areas of 10and 5-cm square were developed for an FRP system, which obtains fine position resolution. These new detectors are useful for high position resolution and transmission experiments with a compact neutron source.

\section{Acknowledgments}

I would like to thank the staff in charge of the RPMT in Hamamatsu Photonics Co. After announcing the discontinuation of the PMT for the RPMT detector, they informed me of the outline of a replacement detector. I greatly appreciate that they lent me the replacement detector prototype on condition that I develop a new readout circuit.

I would like to thank Dr. S. Koizumi in Ibaraki University who lent the FRP4 detector that he bought for the first time. I confirmed that the FRP4 detector has a lower distortion than the RPMT detector, which gave me a chance to develop it in earnest.

I would like to thank Dr. H. Endo and Dr. N. Yamada of KEK who permitted me the use of BL06 and BL16 in the MLF.

Finally, I would like to thank the staff of RIKEN compact neutron source (RANS) and Kyoto University compact neutron source (KUANS). I was able to perform the neutron beam irradiation tests of the FRP detector, although it occurred between experiments of other detectors.

[1] S. Satoh et al., IPS08 Proceedings of the International Symposium on Pulsed Neutron and Muon Sciences at J-PARC 2008, DOI:10.1016/j.nima.2008.11.054.

[2] S. Satoh, "Development of readout systems for neutron detectors," Hamon 15(1), 78-81 (2005), in Japanese.

[3] Hamamatsu Photoniccs, R3292 Datasheet, TPMH1238E01 Sept. 1998. 